\title{
AULA
}

Revista de Pedagogía de la Universidad de Salamanca

ISSN: 02I4-34O2 - e-ISSN: 2I74-0925 - DOI: http://dx.doi.org/IO.I42OI/aula2O2O26

CDU: 37 - IBIC: Educación; Pedagogía (JN) - BIC: Education (JN)

BISAC: EDUCATION / General (EDUOOOOOO)

Vol. 26, 2020

SUMARIO ANALÍTICO

Eva García Redondo

Universidad de Salamanca

Correo-e: evagr@usal.es

\section{La educación de las niñas desde una perspectiva global. Contextos, reflexiones y experiencias}

Resumen: La atención, todavía escueta, que se viene prestando a la educación de las niñas está marcada por un fuerte sentido de necesidad, a la vez que de complejidad. Las circunstancias y contextos que envuelven a su género, a nivel familiar, social, político y religioso, resultan determinantes en el diseño y desarrollo de propuestas pedagógicas. Dado que se ha avanzado más en el estudio y análisis de la niña en el marco de los sistemas educativos, optamos, en el presente artículo, por centrar nuestra atención en el de la educación no formal. Siendo así, reflexionamos en torno a las causas que llevan al colectivo a ser considerado vulnerable y, por ende, prioritario en el devenir de estrategias educativas, a la vez que ofrecemos diversas experiencias que, asentadas sobre preceptos, datos y lógicas ofrecidas por organismos supranacionales (UNESCO, UNICEF...), pretenden arrojar luz para romper con la invisibilidad que, aún a día de hoy, sigue mostrando el colectivo a nivel educativo en muchos contextos.

Palabras clave: educación de las niñas; educación de género; desigualdad; educación no formal.

Pilar Abós Olivares

Departamento de Ciencias de la Educación. Universidad de Zaragoza

Correo-e: pabos@unizar.es

\section{La escuela ubicada en territorios rurales: una escuela diferente, un reto pedagógico}

Resumen: El texto que se presenta analiza cómo y por qué la escuela ubicada en territorios rurales es un modelo de escuela diferente ya que la diversidad de edades, capacidades e intereses es su característica primordial, que la convierte en un espacio educativo en el que la innovación se convierte en un reto pedagógico que permita una 
transformación estructural dirigida a potenciar la inclusión y la justicia social. Partiendo de esta premisa, el artículo aborda el significado de la innovación en el marco de la escuela rural afrontando los procesos innovadores como retos pedagógicos en cuanto a la creación de una escuela abierta y conectada con el territorio, una escuela que valora lo local en el marco de la aldea digital y una escuela inclusiva que enseña a aprender.

De este modo, los elementos que caracterizan a la escuela situada en territorios rurales como una escuela diferente nos conducen a una reflexión crítica sobre los contextos, las prácticas pedagógicas y la relación entre ambos partiendo de la idea de que la diversidad es la referencia para transformar la realidad socioeducativa del siglo xxI.

Palabras clave: escuela ubicada en territorios rurales; diversidad; innovación; inclusión.

\section{Adecir Pozzer}

Secretaria de Estado da Educação de Santa Catarina (SED/sC/Brasil)

Correo-e: pozzeradecir@hotmail.com

Elcio Cecchetti

Universidade Comunitária da Região de Chapecó (UNOCHAPECó/Brasil)

Correo-e: elcio.educ@hotmail.com

\section{Escolas indígenas. Contextos, percepções e desafios interculturais}

Resumo: $\mathrm{O}$ artigo trata da escola indígena no Brasil, seus princípios, objetivos, organização e desafios contemporâneos. Reflete criticamente os aspectos relativos as condições e possibilidades da formação cultural e técnico-profissional dos alunos indígenas, bem como a dimensão epistêmico-metodológica que fundamenta a educação escolar indígena intercultural. Trata-se de uma abordagem qualitativa, cuja metodologia adotada é bibliográfica e documental. Ao analisar os parcos avanços ocorridos nas últimas décadas e os inúmeros desafios ainda existentes, conclui-se que a educação escolar indígena carece de maior autonomia e investimento estatal, de modo que, gradativamente, as próprias comunidades indígenas possam fazer a gestão de suas unidades escolares. Mesmo diante de inúmeras dificuldades, as experiências de educação escolar indígena intercultural tem proporcionado maior reconhecimento, valorização e respeito ao pensamento, formas de vida e ciências produzidas no interior das comunidades indígenas, mesmo elas estando às margens dos sistemas culturais, econômicos e políticos hegemônicos.

Palavras Chave: educação; escolas indígenas; interculturalidade.

M. ${ }^{a}$ Dolores Fernández Malanda

Universidad de Burgos

Correo-e: fermal@ubu.es

Mara García Rodríguez

Universidad de Burgos

Correo-e: maragr@ubu.es 


\title{
Chiri Wayrita: la voz de los sin voz. Una experiencia de educación transformadora para los niños y niñas del Cerro Rico (Bolivia)
}

\begin{abstract}
Resumen: Desde hace una década, la Universidad de Burgos, a través del Vicerrectorado de Internacionalización y Cooperación y el Centro de Cooperación y Acción Solidaria, apuesta por proyectos educativos y de salud en Bolivia, concretamente en el Departamento de Potosí. A lo largo de estos años, estudiantes y profesores de diferentes titulaciones hemos participado de una experiencia que ha cambiado nuestra visión del mundo al acercarnos a culturas que sobreviven al individualismo, la cosificación y la despersonalización, pilares del capitalismo neoliberal salvaje, empoderando y fortaleciendo el sentimiento de comunidad. Convivir con los pueblos andinos, quechuas y aymaras; participar de sus modelos de educación propia en escuelas tan complejas y hermosas como Robertito, edificada en el corazón de una de las minas más terribles del planeta, el Sumaq Orcko, nos ha hecho adquirir una nueva y enriquecedora visión del mundo a partir de una cosmovisión para nosotros reveladora por los principios de solidaridad y reciprocidad sobre los que se ha construido (Fernández Malanda, D. en Cifuentes García y Gómez Campelo, 2016: I2I).
\end{abstract}

Palabras Clave: niños, niñas y adolescentes trabajadores; explotación infantil; mina; Escuela Robertito; protagonismo infantil.

\author{
Daniela Maturana Castillo \\ Universidad de Santiago de Chile \\ Correo-e: daniela.maturana@usach.cl \\ Paulina Fernández SÁNCHEZ \\ Colegio Notre Dame. Santiago de Chile \\ Correo-e:pfernandez@notredame.cl
}

\section{Educar en contexto de segregación territorial. El caso de Bajos de Mena}

Resumen: Explicar el sistema educacional chileno y la educación en contextos de exclusión y desigualdad requiere de un análisis que supere las condiciones educativas y se adentre en un plano de mayor profundidad sobre la segregación. En este artículo se explora la relación entre políticas habitacionales y educacionales implementadas de la mano del sistema neoliberal a partir de la década de 1980 hasta la actualidad en Chile, particularmente en la localidad de Bajos de Mena, Santiago. Considerando estas políticas y su relación en la definición de la desigualdad, se analiza la complejidad que supone educar en estos contextos, a partir del análisis de contenido aplicado al relato de un profesor de una escuela del sector, donde la relación laboral y educativa se condiciona por la violencia y la distancia simbólica entre estudiantes y profesores.

Palabras clave: desigualdad; segregación; exclusión; marginalidad. 
Leoncio VeGA GIL

Universidad de Salamanca

Correo-e:lvg@usal.es

Andrea Martín García

Universidad de Salamanca

Correo-e: andreamartin@usal.es

\section{Las escuelas low cost, sin docentes. La privatización de la educación «por defecto» en países de bajos ingresos}

Resumen: El presente trabajo de investigación pretende realizar un análisis crítico y sociohistórico de la conformación y expansión de los modelos privados de educación, así como de las lógicas de la privatización de la educación en algunos países de bajos niveles de ingresos y de rentas. Son estos contextos los que están siendo utilizados por las nuevas formas de educación para experimentar, asentar y expandir modelos educativos de bajo coste y, por tanto, asequibles para las familias de bajos ingresos en contextos de precariedad económica y social. El trabajo se centra en el análisis de dos países y dos proyectos educativos. Se trata de Kenia, país epicentro de experimentación y expansión del modelo de BIA (Bridge International Academies), y Perú, en el que están proliferando como las margaritas las Innova Schools (IE) de la mano de la corporación industrial y comercial más importante del país. Estos proyectos, apoyados incluso por las instituciones oficiales, son fruto de la ausencia de la red pública a causa de las limitaciones estructurales. La opción privada es más rentable económicamente y de mayor nivel de calidad que la ofrecida por la red oficial. Al final, estos proyectos ni son tan integrados, ni tan sociales, ni tan excelentes, ni tan pedagógicos como se presentan.

Palabras Clave: privatización de la educación; educación por defecto; capitalismo pedagógico; bajo coste.

Ana Yara Postigo Fuentes

Universidad de Málaga

Correo-e: anayaratranslator@gmail.com

Pedro Francisco González RoA

Universidad dos Açores

Correo-e: pedrogonzalez@uac.pt

\section{El Movimiento de la Escuela Moderna de Portugal en el aula de hoy. Un caso práctico}

Resumen: En este artículo se presenta una práctica en la actualidad en el aula del Movimiento de la Escuela Moderna en Portugal. En el día a día se ve reflejada la filosofía detrás del Movimiento y su cultura pedagógica en un contexto particular de aprendizaje en el que la docente titular organiza su trabajo cotidiano siguiendo las líneas de esta propuesta pedagógica. Se ha llevado a cabo una observación etnográfica, entrevista a la docente titular y a una docente en prácticas, además de conversaciones informales con 
ellas, y análisis de documentos de trabajo en el aula para una mayor comprensión de la práctica. Como principales resultados se presentan la actualidad de los principios del Movimiento hoy en día y su planteamiento relevante en el aula.

Palabras Clave: mem; democracia; práctica educativa; participación; autonomía; organización escolar.

Gelson WeschenfeLder

Universidade LaSalle. Canoas, Brasil

Correo-e: gellfilo@gmail.com

\section{Quadrinhos de super-herói em sala de aula}

Resumo: As histórias em quadrinhos podem auxiliar neste gigantesco problema de nossa educação nacional. Elas podem a se tornar objeto de prática de ensino, na tentativa de introduzir o hábito de leitura e de conhecimento nos alunos. Esta pesquisa apresenta a educação através de outras mídias, a importância das histórias em quadrinhos, o seu aspecto pedagógico, principalmente no que tange ao hábito de leitura e à formação da consciência moral das crianças e dos adolescentes e na construção de sua vida. O fascínio que os personagens exercem, fazem o leitor se prender a leitura deste gênero literário, iniciando assim o hábito pela leitura.

Palavras-chave: histórias em quadrinhos; leitura; cultura e mídias na educação.

Roberto RuIz ANDRÉs

Universidad de Burgos

Correo-e: rrandres@ubu.es

\section{Dificultades y contradicciones en la respuesta educativa para los alumnos con necesidad específica de apoyo educativo (ACNEAE)}

Resumen: Se considera que la respuesta educativa que se viene ofreciendo al alumnado con necesidad específica de apoyo educativo en institutos o centros de educación secundaria es insuficiente y claramente mejorable. Tras poner de manifiesto que en dichos centros se vulnera el principio de atención a la diversidad que debe impregnar toda la enseñanza básica, así como el derecho a la plena participación, aprendizaje y éxito de todos que proclama el modelo de escuela inclusiva, en el presente documento se reflexiona sobre cómo la normativa actual clasifica al alumnado que requiere una atención educativa diferente a la ordinaria, estableciendo una marcada barrera conceptual entre los alumnos con necesidades educativas especiales (ACNEE) y el resto de los grupos de alumnos con necesidad específica de apoyo educativo (ACNEAE) que también pueden presentar un desfase significativo de dos o más cursos en el nivel de competencia curricular; mientras que para los primeros se reservan exclusivamente las adaptaciones curriculares significativas (ACS), la respuesta educativa que se prevé para los segundos (ACNEAE que no 
son ACNEE) queda en la más absoluta indefinición, cayendo además en graves incongruencias a la hora de diseñar y desarrollar una propuesta educativa adecuada $\mathrm{y}$, sobre todo, a la hora de abordar una de las partes esenciales del proceso de enseñanza/aprendizaje: la evaluación. En este sentido, sin renunciar por ello a reclamar una trasformación más profunda y estructural del Sistema Educativo, se propone un cambio en la normativa para que en las materias en las que este alumnado recibe apoyo específico, normalmente Lengua y Matemáticas, la evaluación tome como referentes los elementos planteados en las propuestas curriculares individuales que se diseñan a partir de su nivel de competencia curricular.

Palabras clave: respuesta educativa; alumno con necesidad específica de apoyo educativo; necesidades educativas especiales; adaptación curricular significativa.

Antonio Palomino Martín

Correo-e: tpalomino@gmail.com

Eduardo Ramos Verde

Universidad de las Palmas de Gran Canaria

Correo-e: eduardo.ramos@ulpgc.es

\section{La interacción profesorado-alumnado en las clases universitarias, influencia del clima del aula}

Resumen: Con este trabajo estudiamos la interacción que se produce en algunas aulas universitarias entre el profesorado y el alumnado en las distintas ramas de conocimiento. Participaron is profesores y 560 alumnos. Los resultados nos permiten afirmar que hay diferencias considerables entre las categorías de Distancia y Cercanía, mostrando la primera un total de 359 evidencias por 532 de la segunda respectivamente, lo que indica una clara inclinación hacia una interacción positiva entre el profesorado y el alumnado.

Palabras Clave: interacción profesorado-alumnado; aula universitaria; clima del aula.

Antonia Solange Pinheiro Xerez

Universidade Estadual do Ceará (UECE)

Correo-e: antonia.xerez@uece.br

Luís Távora Furtado Ribeiro

Universidade Federal do Ceará (UFC)

Correo-e: luistavora@uol.com.br

Racquel VAlÉRIO MarTins

Instituto de Iberoamérica de la Universidad de Salamanca, USAL

Correo-e: racquelvm@gmail.com

Karla Angélica Silva do Nascimento

Centro Universitário Christus (UNICHRISTUS)

Correo-e: karla.asn@gmail.com 


\section{Jovens da escola pública: diálogos e reflexões acerca das possibilidades e limites de seu ingresso nas universidades brasileiras}

Resumo: O presente estudo tem como objetivo refletir acerca dos limites e possibilidades de acesso do estudante do ensino médio da rede pública brasileira à universidade. Para tanto, por meio das metodologias interativa, dialógica e reflexiva aplicadas no desenvolvimento de um projeto de extensão desenvolvido na Universidade Estadual do Ceará (UECE), foi possível identificar, na fala dos duzentos participantes, compreendendo estudantes e professores do ensino médio, elementos importantes para compreender o ingresso de jovens do Ensino Médio durante todo o processo da referida pesquisa. Assim, tomamos como referencial teórico-metodológico, teorias críticas que buscaram despertar nos jovens, atitudes de formação política, autonomia e emancipação. Constatamos que ainda há um distanciamento entre a universidade e a escola e que as políticas estudantis de acesso e permanência do estudante egresso do ensino médio para ingressar e permanecer na universidade não são amplamente conhecidas pelos estudantes da escola pública de ensino médio do Ceará/Brasil, lócus desta pesquisa.

Palavras-chave: jovens; escola; universidade; política estudantil.

Eugénie EYEANG

CRAAL, École Normale Supérieure (Libreville, Gabon)

Correo-e: eyeangeugenie@gmail.com

\section{Las educaciones africanas a lo largo de los siglos: una navegación entre varios sistemas educativos}

RESUMEN: Ni África es un continente uniforme (podríamos hablar de varias Áfricas), ni tampoco se puede hablar de una sola educación africana, por lo que hemos de hablar en plural. Más aún si nos referimos a la enorme diversidad de pueblos, lenguas y culturas que pueblan el continente de norte a sur y de este a oeste. También parece obvio hablar de diversidad y en plural cuando nos referimos a los sistemas educativos actuales de las diferentes naciones africanas, que construyen sus modelos educativos propios a partir de sus respectivas independencias en el corazón del siglo xx, pero tomando en consideración muchas de las tradiciones africanas ancestrales de los pueblos originarios. En esta confluencia de situaciones se encierra la clave interpretativa del ser educativo del continente africano actual, que posee riquísimas y tradiciones ancestrales, incluso con culturas escritas sincrónicas a las griegas, las judeocristianas primitivas y árabes, y no solo orales, rompiendo así alguno de los tópicos imperantes sobre la ausencia de la cultura escrita entre los africanos. Un análisis comparado de varias muestras y ejemplos nos conduce a una interpretación menos lineal y tradicional de los modelos educativos africanos de nuestro tiempo.

Palabras clave: África, educación; sistemas educativos; culturas africanas originarias. 
Evangelina BonIFÁcIO

Centro de Estudos para o Desenvolvimento Humano (CEDH)

Instituto Politécnico de Bragança/ESE

Correo-e: evangelina@ipb.pt

Isabel BAPTISTA

Centro de Estudos para o Desenvolvimento Humano (CEDH)

Universidade Católica Portuguesa/FEP

Correo-e: ibaptista@porto.ucp.pt

\section{Ética e deliberação prática. As narrativas dos educadores sociais}

Resumo: Este texto apresenta os resultados de parte de um estudo realizado junto de um grupo de educadores sociais portugueses visando compreender de que forma estes técnicos da ação socioeducativa lidam com as questões éticas emergentes do seu quotidiano profissional. Para o efeito, recorreu-se a uma estratégia de investigação assente na recolha de narrativas produzidas pelos próprios atores, em alinhamento com uma conceção de ética prática de teor eminentemente dialógico e relacional. Tal como será sublinhado, os resultados recolhidos e analisados evidenciam necessidades específicas de formação ético-deontológica, com ênfase para as aptidões de deliberação em contexto.

PALAVRAs-chave: educadores sociais; ética socioeducacional; conhecimento profissional; deliberação prática.

Mónica Gallego López

Universidad de Burgos

Correo-e: mgallegol@yahoo.com.mx

\section{Educación y formación de intérpretes del arpa de pedales en Colombia y España en el siglo XXI}

Resumen: Este trabajo brinda un acercamiento a los arpistas de pedales (maestros, intérpretes y alumnos) en Colombia y España, y ofrece una visión de la formación de los arpistas en el siglo xxI. Se realiza un acercamiento cualitativo a los arpistas en la actualidad con el diseño, desarrollo, análisis y evaluación de entrevistas, y participación en importantes eventos musicales, culturales y pedagógicos en torno al arpa de pedales, en los respectivos países. Se ofrecen significativas reflexiones en torno a la educación musical y, en especial, diferentes puntos de vista sobre la formación de los arpistas y el desarrollo profesional y educativo, convirtiéndose en un estudio pionero. La investigación muestra las aplicaciones que de la historiación crítica del arpa pueden derivarse para la formación de arpistas y el reconocimiento de su valor.

Palabras Clave: formación musical; educación; arpistas; desarrollo cultural. 